\title{
THE EFFECT OF ULTRASONIC SCALING ON MICROLEAKAGE AROUND ZIRCONIA CROWN MARGINS LUTIED WITH TWO RESIN CEMENTS
}

\author{
Amir Shoukry Azer*
}

\begin{abstract}
Aim: To evaluate the effect of ultrasonic scaling on microleakage around crown margins after being cemented with two resin cements.

Materials and Methods: Thirty two maxillary premolars were prepared to receive zirconia crowns. Specimens were divided into two groups ( $n=16)$ : Group I, crowns cemented with self-etch adhesive resin cement (Panavia F 2.0), Group II, crowns cemented with self-adhesive resin cement (Panavia SA Cement Plus). Each group was further subdivided into two subgroups according whether or not piezoelectric ultrasonic scaling was performed. All specimens were thermocycled then immersed in basic fuchsine dye before being sectioned buccolingually. Microleakage was then evaluated using the stereomicroscope. Microleakage percentage was determined and statistical comparisons were conducted using ANOVA followed by post hoc test.
\end{abstract}

Results: There was no statistically significant difference in microleakage percentage for zirconia crowns when being cemented with self-etch resin cement (Panavia F 2.0) with or without the application of ultrasonic scaling at the crown margins $\left(\mathrm{P}_{1}=0.017\right)$. However, there was a significant difference in microleakage of specimens cemented with the self-adhesive resin cement (Panavia SA Cement Plus), where higher microleakage percentage was noticed after application of ultrasonic scaling than those without treatment $\left(\mathrm{P}_{2}<0.001\right)$.

Conclusion: Ultrasonic scaling could affect tooth/crown margin interface with subsequent microleakage. Self-etch adhesive resin cement showed higher resistance to ultrasonic scaling with resultant less microleakage compared to self-adhesive resin cement. Both types of resin cement could be used clinically, since the microleakage values of self-adhesive resin cement were within acceptable range compared to other studies.

KEY WORDS: Microleakage, ultrasonic scaling, zirconia crowns, resin cement.

* Lecturer of Fixed Prosthodontics, Faculty of Dentistry, Alexandria University. 


\section{INTRODUCTION}

In recent years, the patients' growing demand for highly natural appearing restorations has influenced the development of new all-ceramic materials with improved mechanical properties guaranteeing suitable durability and limiting technical drawbacks, which are currently substituting conventional metalceramic restorations. ${ }^{(1-3)}$

Zirconia ceramics is a polymorphic and allotropic polycrystalline material fabricated with CADCAM machinery from fully or partially sintered blanks. It illustrates excellent mechanical characteristics compared to metal ceramics. . $^{(3,4)}$

Zirconia is a highly biocompatible material and can prevent crack propagation inducing a significant rise in fracture toughness by means of a recognized mechanism called transformation toughening. Lately, monolithic zirconia has been introduced to the profession with a promising prognosis and has been advocated for all-ceramic fixed partial dentures as well as single crowns. ${ }^{(1,2)}$

Marginal adaptation is a principal factor for long-term longevity and clinical success of dental restorations. ${ }^{(5)}$ Marginal misfit and irregularities may be a reason for cement exposure to oral fluids, consequentially causing marginal microleakage and luting agent dissolution. In such conditions, marginal discrepancies enhance incubation of bacteria, food and oral debris, potentially resulting in secondary caries, marginal discoloration, hypersensitivity and periodontal disease. ${ }^{(6-10)}$

The selection of dental cement is one factor that may affect marginal seal. Several types of cements may affect the marginal seal as they differ based on their polymerization shrinkage, hygroscopic expansion, coefficient of thermal expansion, bond with tooth structure, and bond with zirconia. ${ }^{(11-15)}$

A survey established in 2013, found that 55\% of dentists utilize resin modified glass ionomer (RMGI) cement and 39\% of dentists use a resin cement for zirconia crowns. ${ }^{(2)}$ Several in vitro studies have shown increased microleakage, as measured by dye penetration, around margins of crowns cemented with RMGI cements compared to resin cements. $^{(6,16-19)}$

Recently, the use of resin luting agents is recommended for the cementation of all ceramic systems as it strengthens the restoration ${ }^{(19)}$. Unfortunately these resin luting systems require a multistep application technique, which can be a complex procedure and is reported to be highly sensitive to the operator expertise. ${ }^{(20)}$ Thus, self- adhesive resin cements are now commonly used for the cementation of all-ceramic crowns, in the spirit to simplify the cementation procedure. ${ }^{(21)}$

Sonic and ultrasonic scalers are traditional tools used for removing plaque and calculus from tooth surface intraorally. Ultrasonic scalers are divided into two main systems: magnetostrictive (elliptical vibration pattern active on all edges of the tip) and piezoelectric (linear vibration pattern with only two active edges of the tip). Sonic scalers vibrate between 3.000 and 8.000 cycles per second (Cps), while magnetostrictive and piezoelectric units vibrate between 18.000 and 45.000 Cps and 25.000 to $50.000 \mathrm{Cps}$, respectively. ${ }^{(22-24)}$ It is assumed that the vibrational forces produced by piezoelectric ultrasonic scalers may disrupt the cement bond existing at the crown margin. ${ }^{(24)}$

An in vitro study concluded that more negative effects on surface roughness of resin-based restorative materials was observed on using magnetostrictive ultrasonic scalers than sonic scalers ${ }^{(23)}$. Piezoelectric ultrasonic scalers are clinically preferable owing to their quieter operation, smaller tips and handpieces, and ease of use. ${ }^{(24)}$ It was shown that ultrasonic scaling with a piezoelectric unit led to microleakage at the cementum margin of class $\mathrm{V}$ restorations. It was thus concluded that ultrasonic scaling might cause disruption of the resin bond leading to microleakage. ${ }^{(25-27)}$ 
The purpose of this in vitro study was to compare the microleakage around full-zirconia crown margins luted with two resin cements after ultrasonic scaling. The null hypothesis was that there would be no difference in terms of microleakage around zirconia crown margins either before or after ultrasonic scaling when using the two tested resin cements.

\section{MATERIALS AND METHODS}

Thirty two human maxillary premolars of nearly the same size (average crown dimensions of 8.5 $\mathrm{mm}$ cervico-occlusally and $7 \mathrm{~mm}$ mesio-distally) were collected from the Oral Surgery Department, Alexandria University. All teeth were examined for caries by the aid of a sharp explorer. Any teeth presented with crack, fracture lines or caries were excluded from this study.

All teeth were stored in $0.5 \%$ chloramine $\mathrm{T}$ solution at $4^{\circ} \mathrm{C}$ immediately after extraction to prevent bacterial growth. Teeth were notched at the roots and embedded into an acrylic resin base to facilitate teeth preparation and future crown cementation.

Only one experienced operator performed almost identical full crown preparations to receive single crowns, with approximately $1.5 \mathrm{~mm}$ occlusal reduction and an axial reduction of $1.5 \mathrm{~mm}$ with approximately $12^{\circ}$ taper. The cervical preparation margin thickness was $1.0 \mathrm{~mm}$ circumferential rounded chamfer $0.5 \mathrm{~mm}$ coronal to the $\mathrm{CEJ}^{(27)}$ (Figure 1).

All prepared teeth were scanned using the CEREC AC Omnicam (Sirona Dental Systems, Bensheim, Germany), followed by designing the full-anatomic thirty two crowns using CEREC 3D software (version 4.2, Sirona Dental Systems, Bensheim, Germany). All crowns were of the same design and were enlarged by $30 \%$ for milling in a partially sintered state. A CEREC inLab MC XL milling unit was used for the CAM process of the designed crowns using In Coris TZI zirconia blocks. This was followed by sintering cycle by gradual rise in temperature till it reached $1540^{\circ} \mathrm{C}$. The overall sintering time was about six hours. Zirconia crowns were seated on their corresponding prepared teeth to verify their fit. Prior to cement application, all zirconia crowns were abraded with $50 \mu \mathrm{m}$ aluminum oxide air born particles at 2 bar pressure, and cleaned for $5 \mathrm{~min}$ in an ultrasonic water bath as recommended by the manufacturer.

Teeth and their respective crowns were randomly divided into two main test groups. Group I $(n=16)$ : Crowns cemented using Panavia F 2.0 (self-etch adhesive resin cement, Kuraray Dental, Japan) Group II ( $\mathrm{n}=16)$ : Crowns cemented using Panavia SA Cement Plus (self-adhesive resin cement, Kuraray Dental, Japan).

Cementation procedure was done according to the manufacturer's recommendations. Each specimen was put under a static load cementing device of $5 \mathrm{~kg}$ to ensure constant pressure during cementation. Restoration's margin was light cured for 5 seconds at each surface, and excess cement was removed around the marginal interface. The cement was continued to polymerize by light cure for 25 seconds at each margin.

For ultrasonic waves application, each group was further subdivided into two subgroups: Group I $(n=16)$ was divided into subgroup Ia $(n=8)$ a control group with no ultrasonic application and subgroup Ib $(n=8)$ in which specimens were subjected to ultrasonic waves. Similarly, group II $(n=16)$ was divided into subgroup IIa $(\mathrm{n}=8)$ as a control and IIb $(n=8)$ for ultrasonic application.

Ultrasonic scaling with piezoelectric device (UDS-K Ultrasonic piezo scaler, Guilin Woodpecker medical instrument Co. LTD China) was utilized at full power with distilled water. The lateral side of the tip was used to trace the crown-dentin interface for 60 seconds under moderate hand pressure on each side of the crowns (Figure 2). 
Specimens were subjected to thermocycling between 5 and $55^{\circ} \mathrm{C}$ for a total of 500 cycles. The teeth were then immersed in 5\% basic fuchsine dye (Fischer Scientific Company, Fairlawn, NJ) solution for 24 hours. Specimens were then rinsed thoroughly in water, embedded in clear acrylic resin blocks and sectioned buccolingually using a diamond coated thin laboratory disc. (Jelenko, Armonk, NY, USA)

All specimens were examined under stereomicroscope (Olympus SZ-CTV, Japan) at 30x magnification for dye penetration from the external crown surface to the point where no purple dye

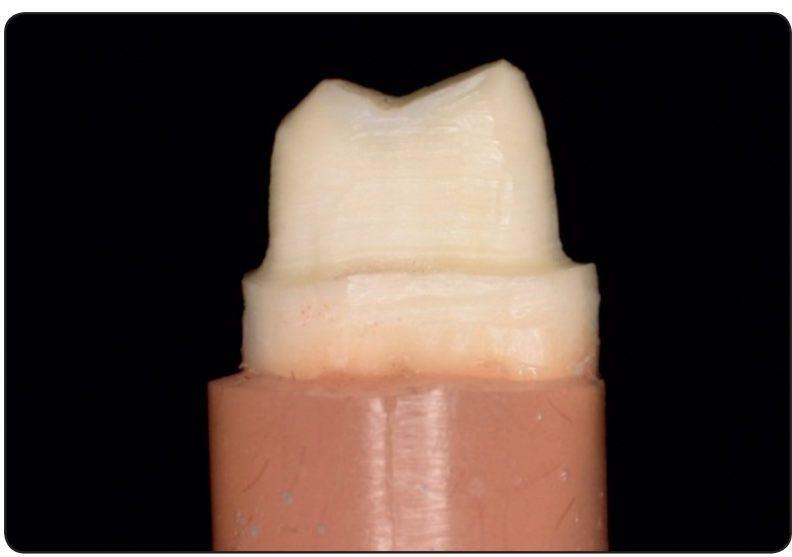

Fig. (1): Prepared tooth for full crown restoration with a $12^{\circ}$ taper and chamfer finish line.

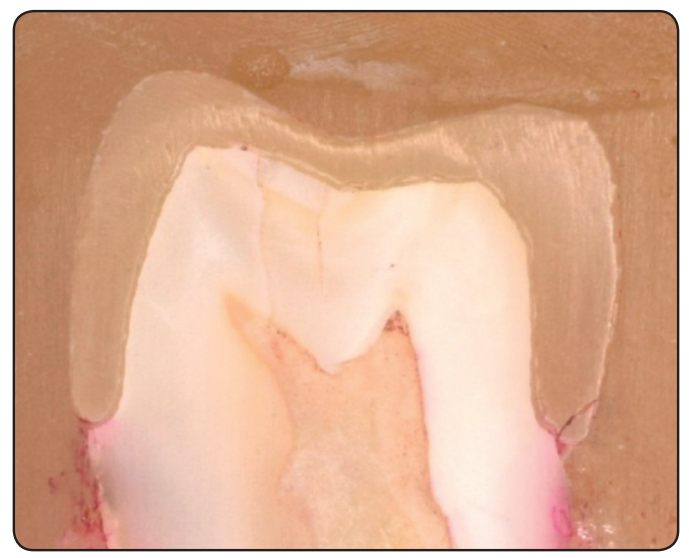

Fig. (3): Sectioned tooth specimen (30 x), showing dye penetration due to microleakage when using Panavia F 2.0 resin cement before applciation of ultrasonic scaling. could be seen, which was measured using an image analysis software (Analysis Starter, Soft Image System, GmbH, Germany) to assign microleakage at both buccal and lingual aspects of each section. Percentage microleakage was measured by dividing the linear distance of dye penetration in microns by the linear distance from the external margin to the axial-occlusal line angle ${ }^{(28)}$ (Figures 3-6).

Descriptive statistics (mean and standard deviation) were used to summarize the amount of microleakage $\%$ for specimens. Data collected from all four studied subgroups were analyzed using IBM

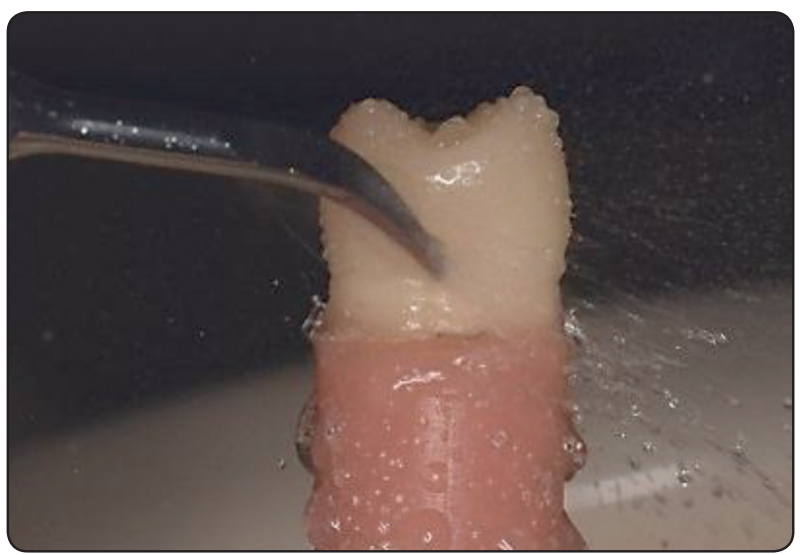

Fig. (2): Ultrasonic scaling of crown margin for 60 seconds.

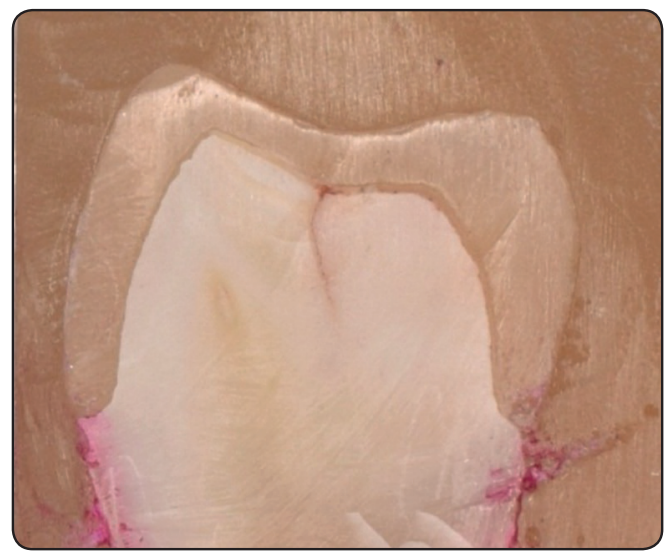

Fig. (4): Sectioned tooth specimen $(30 \mathrm{x})$, showing dye penetration due to microleakage when using Panavia F 2.0 resin cement after application of ultrasonic scaling. 


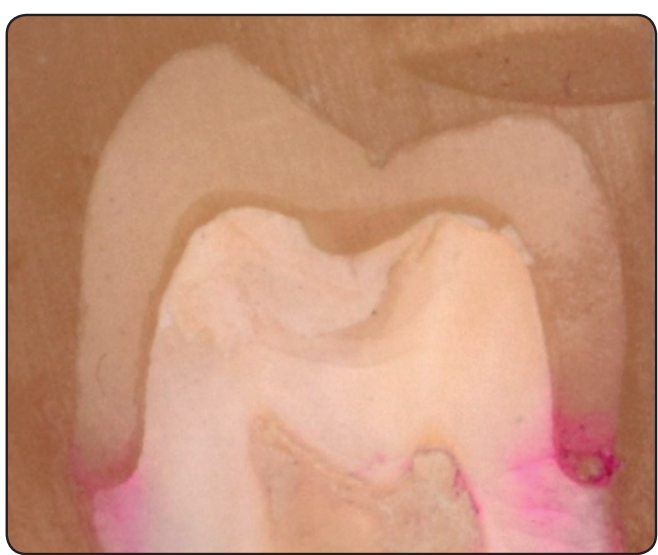

Fig. (5): Secioned tooth specimen (30x), showing dye penetration due to microleakage when using Panavia SA Cement Plus resin cement before applciation of ultrasonic scaling

SPSS software package version 20.0 (Armonk, NY: IBM Corp.). The Kolmogorov- Smirnov test was used to verify the normality of distribution of variables, while Two way ANOVA was used to compare between the different subgroups followed by post hoc test (Tukey-Kramer multiple comparison test).

\section{RESULTS}

The mean values and standard deviations of the four tested subgroups are illustrated in (Table I and Figure 7). In group I, specimens with crowns cemented using self-etch adhesive resin cement (Panavia F 2.0) showed a mean of microleakage percentage and standard deviation of $10.8 \pm 2.3$ for the control (subgroup Ia) and 16.3 \pm 2.4 for the ultrasonically scaled teeth (subgroup Ib). Similarly, In group II $(n=16)$, crowns cemented using selfadhesive resin cement (Panavia SA Cement Plus) showed a mean and standard deviation of $15.4 \pm 2.5$ and $29.8 \pm 5.5$ for the control (subgroup IIa) and ultrasonically scaled teeth (subgroup IIb) respectively.

A two-way ANOVA followed by the Tukey's post hoc paired group comparison procedure of microleakage percentages among the four tested

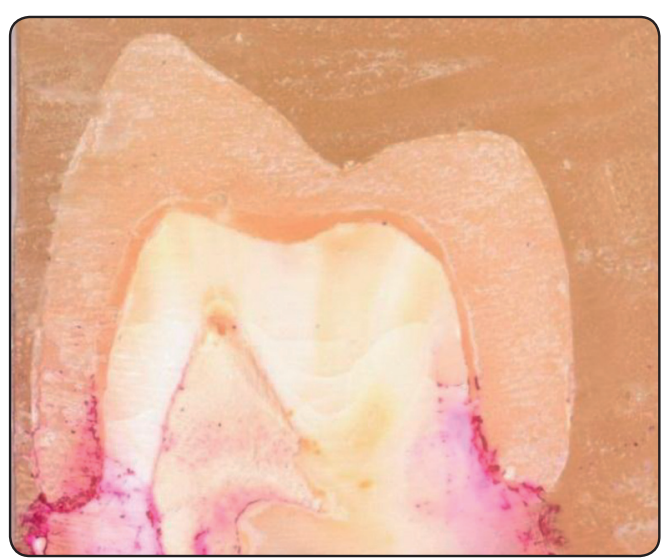

Fig. (6): Secontioned tooth specimen (30x), showing dye penetration due to microleakage when using Panavia SA Cement Plus resin cement after applciation of ultrasonic scaling.

subgroups, revealed no statistically significant difference in microleakage between subgroup Ia and subgroup $\mathrm{Ib}\left(\mathrm{P}_{1}=0.017\right)$. There was a significant difference on comparing the microleakage percentage values between subgroups IIa and IIb $\left(\mathrm{P}_{2}<0.001\right)$, where crown specimens cemented with Panavia SA Cement Plus and subjected to ultrasonic scaling showed higher microleakage values than those without scaling (control subgroup).

Furthermore, there was no significant difference between subgroups Ia and IIa $\left(\mathrm{P}_{3}=0.058\right)$, crown specimens cemented with Panavia F 2.0 and those cemented with Panavia SA Cement Plus, respectively, before application of ultrasonic scaling.

Finally, comparison between microleakage percentage of specimens luted by the two resin cements and after being ultrasonically scaled at crown margins (subgroups $\mathrm{Ib}$ and $\mathrm{IIb}$ ), showed a significant difference $\left(\mathrm{P}_{4}<0.001\right)$, where scaled crown margins of specimens cemented with Panavia SA Cement Plus exhibited a significantly higher microleakage than those cemented with Panavia F 2.0. 
TABLE (1): Comparison between the four studied subgroups according to Microleakage \%

\begin{tabular}{|c|c|c|c|c|c|c|}
\hline \multirow{2}{*}{$\begin{array}{c}\text { Microleahage } \\
\%\end{array}$} & \multicolumn{2}{|c|}{ Group I $(n=16)$} & \multicolumn{2}{|c|}{ Group II (n=16) } & \multirow{2}{*}{$\mathbf{F}$} & \multirow{2}{*}{$\mathbf{P}$} \\
\hline & Ia $(n=8)$ & Ib $(n=8)$ & IIa $(n=8)$ & IIb $(n=8)$ & & \\
\hline Min. - Max. & $6.6-13.3$ & $13.3-20.5$ & $12.2-20.1$ & $20.2-36.2$ & \multirow{2}{*}{$44.723^{*}$} & \multirow{2}{*}{$<0.001^{*}$} \\
\hline Mean \pm SD & $10.8 \pm 2.3$ & $16.3 \pm 2.4$ & $15.4 \pm 2.5$ & $29.8 \pm 5.5$ & & \\
\hline Sig. bet. grps & \multicolumn{2}{|c|}{$P_{1}=0.017$, } & \multicolumn{2}{|c|}{$\mathrm{P}_{4}<0.001^{*}$} & & \\
\hline
\end{tabular}

F: F for ANOVA test, Pairwise comparison between each 2 groups was done using Post Hoc Test (Tukey)

$P$ : $p$ value for comparing between the studied subgroups, where:

P1: p value for comparing between Ia and Ib, P2: p value for comparing between IIa and IIb

P3: p value for comparing between Ia and IIa, $\quad$ P4: p value for comparing between Ib and IIb

*: Statistically significant at $p \leq 0.05$

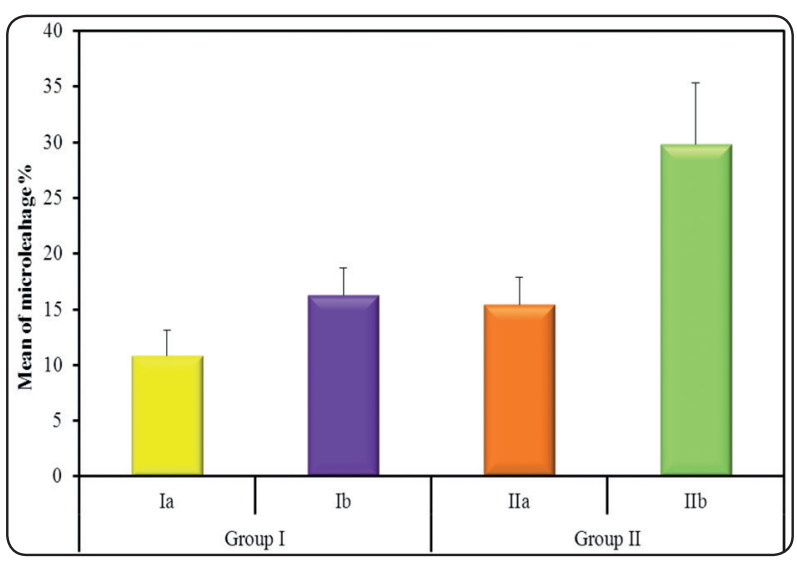

Fig. (7): Comparison between the four studied subgroups according to Microleakage \%

\section{DISCUSSION}

The null hypothesis was partially accepted. Based on the results of this study, there was no statistically significant difference in microleakage percentage of zirconia crowns when being cemented with self-etch adhesive resin cement (Panavia $\mathrm{F}$ 2.0 ) with or without the application of piezoelectric ultrasonic scaling at the crown margins. However, there was a significant difference in microleakage of specimens cemented with the self-adhesive resin cement (Panavia SA Cement Plus), where higher microleakage was manifested after application of ultrasonic scaling than those without treatment (control subgroup).
In the present study, only two types of resin cements were used to test microleakage, due to the fact that bond strength between resin composite and dentin is proved to be the highest among all cements. ${ }^{(6)}$ Furthermore, it was considered that these two tested cements should contain 10-methacryloyloxydecyl dihydrogen phosphate (MDP) for better bonding to zirconia.

All crown margins in the current study were established on enamel, since higher microleakage was observed at a resin-cementum interfaces especially when subjected to ultrasonic scaling as mentioned by Goldstein et al. ${ }^{(19)}$

Ultrasonic scaling was suggested as it is predicted to create marginal microleakage of crowns due to increased mechanical stimulation and disintegration of the cement layer. Moreover, there was no significant difference in the effect of ultrasonic scaling and hand instrumentation on marginal microleakage as described by Rohani et al. ${ }^{(25)}$

The protocol conducted in the present study was an in vitro investigation, in contrast to an old previous in vivo study conducted by White et al, ${ }^{(26)}$ who applied periodontal therapy for six months on hopeless test teeth that received crowns. After which, teeth were extracted and studied for marginal microleakage. So, in spite that the current test 
methodology provided consistent and standardized variables, it lacked others found in the oral cavity such as occlusal forces, presence of saliva, $\mathrm{pH}$ changes and presence of different bacterial strains.

All specimens were subjected to thermocycling between $5^{\circ} \mathrm{C}$ and $55^{\circ} \mathrm{C}$ for a total of 500 cycles in order to simulate temperature changes found in the oral cavity. Such extreme in temperature changes may cause mechanical stresses on the crown margins due to the differences in coefficient of thermal expansion related to the restoration materials, cements and natural tooth structure. ${ }^{(7)}$

Different in vitro methods have been employed to evaluate microleakage around restorations in literature, among which is the use of dye tracer. This was followed by detecting the percentage microleakage rather than the inaccurate scoring system used previously in many studies ${ }^{(7)}$.

There were no similar studies in the literature comparing the effect of ultrasonic scaling on microleakage of teeth restored with full zirconia crowns using only two different resin cements. However, several previous studies compared microleakage when using different types of cements, and reported that resin modified glass ionomer cements showed higher microleakage than resin cements. ${ }^{(7,19,26,28)}$ This was attributed to the higher bond between dentin surface and the resin cement as mentioned by the authors. Furthermore, Chazine et al ${ }^{(10)}$ compared the microleakage around three different all-ceramic crowns and concluded that the marginal fit and cement thickness can be considered less important than the cement bonding at the margins.

In the present study, there was a higher microleakage percentage when using the selfadhesive resin cement and on application of ultrasonic scaling compared to the use of selfetch adhesive resin cement even after ultrasonic application. This could be explained by the superior bonding of the self-etch adhesive resin cement and the use of a separate self-etching primer and bonding procedures on dentinal surface as compared to the self-adhesive resin cement. This was observed also on examining the sectioned crowns, where the dye stain was more intense at the resin cement/tooth interface, which proved that group II specimens expressed higher microleakage percentage than those of group I. This was in accordance with the findings of Chang et al. ${ }^{(28)}$

Furthermore, when comparing the results of each group alone, there was a higher microleakage percentage after application of ultrasonic scaling especially when using the self-adhesive resin cement, though this was not significantly observed in case of self-etch adhesive resin cement group. This proves that piezoelectric ultrasonic scaling may affect and disrupt the bond formed at any restoration/tooth interface. This was in agreement with the findings of several authors. ${ }^{(19,25,28)}$ However, the values of microleakage for the self-adhesive resin cement reported in this current study, showed lower values as compared to other previous studies. ${ }^{(6,28)}$ This might be due to the use of cements supplied from different companies and therefore may contribute to varying bonding to dentin.

While in vitro testing might be limited in its ability to predict clinical performance, the results of this study may provide useful information to help clinicians choose a resin cement material that offers best resistance for microleakage as compared to other types of cements. This was in agreement to other studies which compared a single resin cement to other types of luting cements. ${ }^{(6,10,11,17,26,28)}$

\section{CONCLUSION}

Within the limitations of this study, it can be concluded that:

1- Piezoelectric ultrasonic scaling could affect tooth/crown margin interface with subsequent microleakage. 
2- Self-etch adhesive resin cement showed higher resistance to ultrasonic scaling with resultant less microleakage compared to self-adhesive resin cement.

3- Both types of resin cement could be used clinically, since the microleakage values of selfadhesive resin cement was within acceptable range compared to those of other studies.

\section{REFERENCES}

1. Makhija SK, Lawson NC, Gilbert GH. Dentist material selection for single-unit crowns: findings from the National Dental Practice- Based Research Network. J dent. 2016;55:40-47.

2. Christensen GJ. How to prepare zirconia and IPS e.max rstorations for cementation. Clin Rep. 2013;6:1-3.

3. Christensen GJ. Is the rush to all-ceramic crowns justified? J Am Dent Assoc. 2014;145(2):192-194.

4. Freire Y, Gonzalo E, Lopez- Suarez C, et al. The marginal fit of CAD/ CAM monolithic ceramic and metal-ceramic crowns. J Prosthodont. 2017. https://doi.org/10.1111/ jopr.12590.(Epub ahead of print)

5. Heintze SD. Systematic reviews: I. The correlation between laboratory tests on marginal quality and bond strength. II. The correlation between marginal quality and clinical outcome. J Adhes Dent. 2007;9:77-106.

6. Albert FE, El-Mowafy OM. Marginal adaptation and microleakage of Procera Allceram crowns with four cements. Int J Prosthodont. 2004;17(5):529-535.

7. Schmid- Schwap M, Graf A, Preinerstorfer A, et al. Microleakage after thermocycling of cemented crowns- A meta-analysis. Dent Mater. 2011;27(9):855-869.

8. NgJ, Ruse D, Wyatt C. A comparison of the marginal fit of crowns fabricated with digital and conventional methods. J Prosthet Dent. 2014;112(3):555-560.

9. Ortega R, Gonzalo E, Gomez-Polo M, et al. SEM evaluation of the precision of fit of CAD/CAM zirconia and metal-ceramic posterior crowns. Dent Mater J. 2017;36(4):387-393.

10. Chazine M, Casucci A, Mazzoni A, et al. interfacial nanoleakage and internal cement thickness of three esthetic crown systems. Dent Mater. 2012;28(10):1105-1111.
11. Yüksel E, Zaimoglu A. Influence of marginal fit and cement types on microleakage of all-ceramic crown systems. Braz Oral Res. 2011;25(3):261-266.

12. Sailer I, Makarov NA, Thoma DS, et al. All-ceramic or metal-ceramic tooth supported fixed dental prostheses (FDPs)? A systermatic review of the survival and complication rates. Part I: Single crowns (SCs). Dent Mater. 2015;31(6):603-623.

13. Sidhu SK, Carrick TE, McCabe JF. Temperature mediated coefficient of dimensional change of dental tooth-colored restorative materials. Dent Mater. 2004;20(5):435-440.

14. Sulter EA, Watson LE, Tantbirojn D, et al. Effective expansion: balance between shrinkage and hygroscopic expansion. J Dent Res. 2016;95(5):543-549.

15. Prylinska-Czyzewska A, Piotrowski P, Prylinski M, et al. Various cements and their effects on bond strength of zirconia ceramic to enamel and dentin. Int J Prosthodont. 2015;28(3):279-281.

16. Turker SB, Ozcan M, Mandali G, et al. Bond strength and stability of 3 luting systems on a zirconia dentin complex. Gen dent. 2013;61(7):e10-e13.

17. Rossetti PH, do Valle AL, de Carvalho RM, et al. Correlation between margin fit and microleakage in complete crowns cemented with three luting agents. J Appl Oral Sci. 2008;16(1):64-69.

18. Nakamura K, Mouhat M, Nergard JM, et al. Effect of cements on fracture resistance of monolithic zirconia crowns. Acta Biomater Odontol Scand. 2016;2(1):12-19.

19. Goldstein RE, Lamba S, Lawson NC. Microleakage around class V composite restorations after ultrasonic scaling and sonic tooth brushing around their margin. J Esthet Restor Dent. 2017;29(1):41-48.

20. Bernal G, Jones RM, Brown DT, Munoz CA, Goodacre CJ. The effect of finish line form and luting agent on the breaking strength of Dicor crowns. Int J Prosthodont. 1993;6 (May-June (3)):286-90

21. Viotti RG, Kasaz A, Pena CE, Alexander RS, Arrais CA, Reis AF. Microtensile bond strength of new self-adhesive luting agents and conventional multistep systems. J Prosthet Dent. 2009;102 (November (5)):306-12.

22. Angerame D, Sorrentino R, Cettolin D, Zarone F. The effects of scaling and root planning on the marginal gap and microleakage of indirect composite crowns prepared with different finish lines: An in vitro study. Oper Dent. 2012;37(6):650-659. 
23. Lai YL, lin YC, Chang CS, Lee SY. Effects of sonic and ultrasonic scaling on the surface roughness of toothcolored restorative materials for cervical lesions. Oper Dent. 2007;32(3):273-278.

24. Arabaci T, Cicek Y, Canakci CF. Sonic and ultrasonic scalers in periodontal treatment: a review. Int J Dent Hyg. 2007; 5(1):2-12.

25. Rohani B, Barekatain M, Farhad SZ, et al. Influence of hand instrumentation and ultrasonic scaling on the microleakage of various cervical restorations: an in vitro study. J Contemp Dent Pract. 2017;18(6):437-442.
26. White SN, Yu Z, Tom JF, Sangsurasak S. In vivo microleakage of luting cements for cast crowns. J Prosthet Dent. 1994;71(4):333-338.

27. Lyons KM, Rodda JC, Hood JA. Use of a pressure chamber to compare microleakage of three luting agents. Int J Prosthodont. 1997;10(5):426-433.

28. Chang B, Goldstein R, Lin CP, Byreddy S, Lawson NC. Microleakage around zirconia crown margins after ultrasonic scaling with self-adhesive resin or resin modified glass ionomer cement. J Esthet Restor Dent. 2018; 30: 73-80. 